\title{
Quantitative Mass Spectrometric Identification of Isomers Applying Coherent Laser Control
}

\author{
Johanna M. Dela Cruz, Vadim V. Lozovoy, and Marcos Dantus* \\ Department of Chemistry and Department of Physics and Astronomy, Michigan State University, \\ East Lansing, Michigan 48824
}

Received: July 18, 2005; In Final Form: August 17, 2005

\begin{abstract}
Mass spectrometry (MS) is one of the oldest and most trusted analytical methods for chemical identification. Advances in biology, such as metabolic analysis and proteomics, have fueled a growing number of refinements in this method. Unfortunately, isomers, for example, $o$ - and $p$-xylene, are seldom identifiable by MS because they produce identical spectra. Time-consuming and less sensitive multidimensional methods are subsequently required for structural determination. The sensitivity of MS coupled with shaped femtosecond laser pulses that control molecular fragmentation and ionization results in a new, fast, and reproducible method for molecular identification which is used here to distinguish positional and geometric isomer compounds and quantify their relative concentration in mixtures.
\end{abstract}

Standard mass spectrometry (MS) uses an electron gun to cause electron impact ionization of the analyte. In most cases, this method of ionization is not capable of showing sufficient differences to be used for reliable isomer identification. For subsecond trace analysis of compounds such as isomeric species in mixtures, simple solutions such as gas chromatography (GC)MS are not acceptable. The pursuit of laser-based molecular recognition in our group led us to replace the electron gun and the GC column with a femtosecond laser. By phase shaping the laser pulses, we amplify the sensitivity of the laser-matter interactions, highlighting the differences in their electronic and molecular structures. ${ }^{1}$ The resulting coherent laser control (CLC)-MS method can identify positional and geometric isomers and quantify their relative concentration in isomeric mixtures in less than $1 \mathrm{~s}$.

Femtosecond lasers have long been used as a source of ions in MS; in most cases, these studies were aimed at measuring ultrafast photodissociation dynamics. ${ }^{2,3}$ The energy deposited by the laser pulse on the molecules sets off a number of competing processes - primarily ionization, fragmentation, and redistribution of the energy among the different degrees of freedom in the molecule. The relative yield of the resulting fragment ions can be controlled by shaping the femtosecond laser pulses. ${ }^{4,5}$ When all of the frequencies in the spectrum are in step, all having the same phase value, the pulse achieves its shortest duration and it is said to be transform limited (TL). Specific phase functions that determine the relative phase of different frequency components in the pulse can be introduced using a pulse shaper. ${ }^{6}$ The determination of phase functions that could achieve the desired isomeric discrimination is a problem amenable to computer learning algorithms directed by experimental measurements, as suggested by Judson and Rabitz. ${ }^{7}$

The laser-induced ionization and fragmentation process taking place is multiphotonic in nature. On the basis of the fundamental

* Corresponding author. E-mail: dantus@msu.edu. observations of multiphoton intrapulse interference ${ }^{8,9}$ and the great efficiencies gained using binary phase functions, ${ }^{10}$ an exhaustive evaluation of an entire subclass of phase functions is possible in a practical time scale of less than an hour. The results obtained are then ranked to provide the phase function that yields the largest difference between the mass spectra of the isomers in question. It is this optimal phase that is then used to analyze mixtures of isomers in a fraction of a second. We apply CLC-MS for the identification of the positional (ortho, meta, and para) isomers of xylene, cresol, and nitrotoluene and the geometric (cis and trans) isomers of 3-heptene and 4-methyl2-pentene. The most challenging of these experiments, $o$ - and $\mathrm{p}$-xylene, is outlined here in detail, including the quantitative measurement of their relative concentrations in mixtures. ${ }^{11}$

The standard mass spectrum of $o$-xylene is virtually identical to that obtained for $p$-xylene. ${ }^{12}$ Other analytical methods including infrared, Raman, and nuclear magnetic resonance spectroscopy are successful at identifying these two isomers. However, the detection limit for MS is undoubtedly orders of magnitude lower than these spectroscopic techniques, with the possibility for achieving single ion sensitivity. Another significant asset of MS is its capability of handling complex mixtures by $\mathrm{GC}-\mathrm{MS}^{13}$ and the more recent tandem MS, ${ }^{14}$ but these techniques are more time-consuming. The separation of xylene isomers by GC-MS relies on small differences in retention times and waiting times of several minutes ${ }^{15}$ and requires the running of standards. Isomeric differentiation of xylenes has been achieved via selective self-ion/molecule reactions and tandem MS in ion trap mass spectrometers, but the whole process takes several minutes. ${ }^{16,17}$ Resonance enhanced multiphoton ionization employing molecular cooling in a supersonic beam has also been used to separate xylene isomers, although a tunable UV laser source and a special coupling of a GC column and a supersonic beam valve are necessary. ${ }^{18}$ Chemical reactivity has been coupled with MS for xylene identification, ${ }^{19,20}$ though this approach is not universal.

(C) 2005 American Chemical Society 


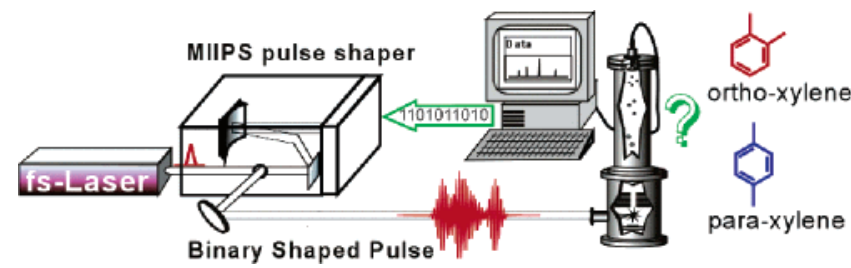

Figure 1. Schematic experimental setup for coherent laser control mass spectrometry for the identification of isomers. The amplified femtosecond laser is sent to a pulse shaper that is programmed to measure and compensate any phase distortions present on the laser pulse. Binary phase functions are introduced at the Fourier plane, where the spectrum of the pulse is fully dispersed. The phase shaped laser pulses are focused inside a low pressure gas chamber where they interact with the sample molecules, causing fragmentation and ionization. A time-of-flight mass spectrometer is used to record the arrival of the ions according to their mass-to-charge ratio. Some binary phase functions cause large enough differences in the fragmentation and ionization patterns that can be used to identify isomers and even quantify their concentration in a mixture.

Previous work from our group, which combined femtosecond pulse shaping with MS, has demonstrated the identification of a number of chemical compounds on the basis of the unique spectral fingerprints generated from different binary phase shaped pulses. ${ }^{1}$ Here, we achieve fast (subsecond) and reproducible isomeric identification of xylene, cresol, nitrotoluene, 3-heptene, and 4-methyl-2-pentene with the experimental setup depicted in Figure 1. Experiments were performed using an amplified 40 fs laser system that was directed to a pulse shaper 6 which was programmed for self-calibration, pulse characterization, and compensation of unwanted phase distortions using the multiphoton intrapulse interference phase scan (MIIPS) method. ${ }^{21,22}$ TL pulses were consequently delivered at the sample. Starting with TL pulses is a critical step for experimental precision and reproducibility because the highly nonlinear interaction between the laser pulses and the molecules, which leads to ionization and fragmentation, is very sensitive to phase variations in the laser pulse. The pulse shaper sequentially introduces predetermined binary shaped pulses. Each function contains 10 groups of 10 pixels. A phase retardation equal to 0 or $\pi$ is assigned to the 10 corresponding regions in the spectrum of the pulse. Because only the phase of the pulse is modulated, the energy and the spectrum of the excitation laser field stay unaltered throughout the experiment.

Laser excitation causes the formation of fragment ions which are then detected by time-of-flight (TOF)-MS. ${ }^{23}$ The mass spectrum obtained for each neat sample was analyzed on the basis of the ratio between the peak value obtained for the molecular ion $\left(\mathrm{M}^{+}, m / z=106\right)$ and the tropylium ion ${ }^{24}\left(\mathrm{~T}^{+}\right.$, $m / z=91)$. A total of 1024 different binary phases representing all possible 10-bit binary phase functions were evaluated. ${ }^{25}$ In Figure 2, we show typical mass spectra obtained for $o$ - and $p$-xylene using CLC-MS for TL pulses (BP0) and for binary phase shaped pulses (BP858). The relative yield of tropylium ion is $130 \%$ for $\mathrm{BP} 0$. The largest relative yield measured for 10-bit binary functions was $210 \%$ for BP858. The differences observed for the other major fragment ions were not as significant, and we did not analyze them further.

Figure 3 shows the statistical distribution of the ratios between the molecular ion and the tropylium ion obtained for each isomer, highlighting the contrast achieved using binary shaped pulses. The ratios are represented by histograms of 100 independent measurements for each sample and for each phase. For TL pulses, a difference between the two isomers is clear. However, the difference observed for BP858 is much greater.
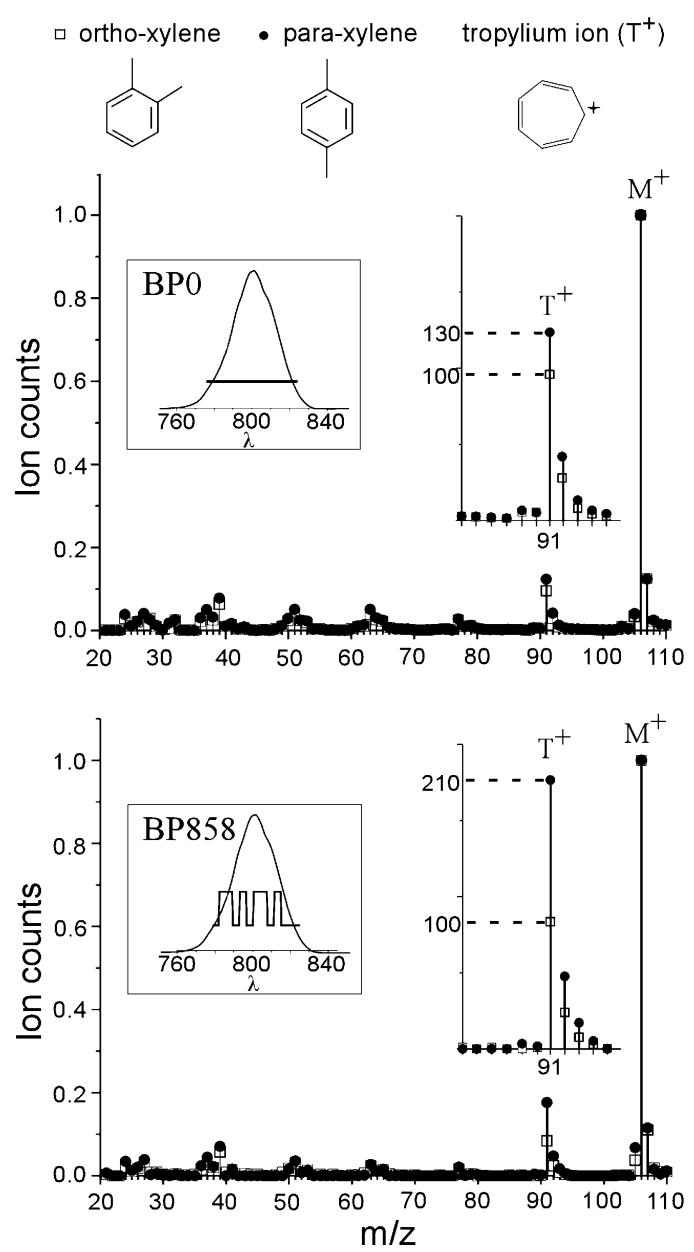

Figure 2. Coherent laser control mass spectra obtained for $o$ - (open squares) and $p$-xylene (filled circles). The top panel shows results obtained with BP0 corresponding to TL laser pulses; the flat phase across the spectrum of the laser pulse is shown in the inset. The bottom panel shows data obtained with laser pulses with binary phase BP858, which is shown together with the spectrum of the pulse in the bottom inset. The mass spectra were normalized so that the molecular ion $\left(\mathbf{M}^{+}\right)$ intensity equals unity. In the top and bottom panels, the region for $\mathrm{m} / \mathrm{z}$ near 91, corresponding to the tropylium ion $\left(\mathrm{T}^{+}\right)$, was amplified to highlight the observed differences. For the BP858 shaped pulses, the relative yield of $\mathrm{T}^{+}$for $p$-xylene is 2.1 times greater than that observed for $o$-xylene.

It is of fundamental interest to understand the mechanism responsible for the observed laser-based recognition. The nonlinear optical polarizability of molecules is highly dependent on molecular structure. ${ }^{26}$ Strong laser fields have been found to cause charge buildup at the ends of extended conjugated systems resulting in ionization by tunneling or barrier suppression. ${ }^{27}$ A greater nonlinear optical polarizability, in this case, that of $p$-xylene, increases a molecule's susceptibility to undergo fragmentation. This is consistent with our results and with the slightly lower ionization energy of $p$-xylene $(8.45 \mathrm{eV})$ compared to that of $o$-xylene $(8.56 \mathrm{eV})$. In Figures 2 and 3, we compare the histograms obtained for BP0 and BP858 to find that, for $p$-xylene, the shaped pulse enhances fragmentation, leading to a decrease in the $\mathrm{M}^{+} / \mathrm{T}^{+}$ratio. Interestingly, the opposite trend is observed for $o$-xylene. The temporal profiles for the two different laser pulses used in Figures 2 and 3 are very different, as shown in the insets of Figure 3. The total ion yield was about 6 times lower for BP858. Analysis of all possible 1024 different shaped pulses led us to conclude that neither the time duration nor the total integrated second harmonic generation of the shaped pulses correlated with the relative yield of the tropylium ion. 


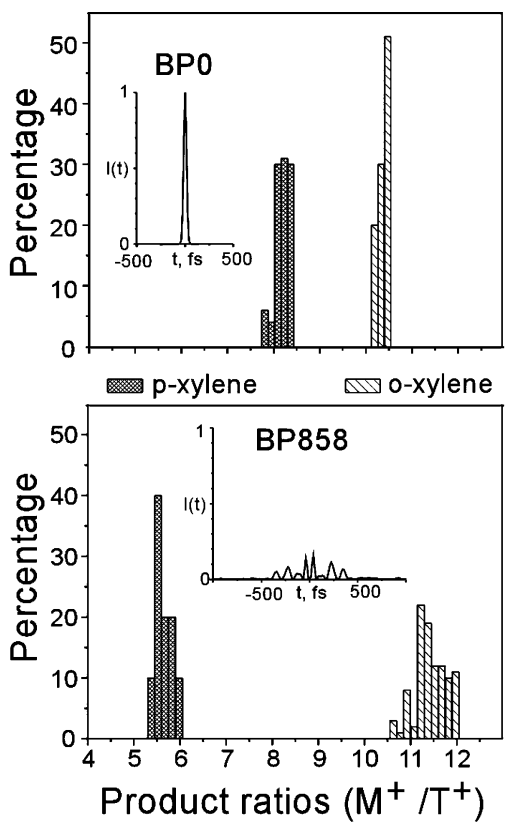

Figure 3. Histograms showing the statistical distribution of the experimentally measured ratio $\left(\mathrm{M}^{+} / \mathrm{T}^{+}\right)$obtained for 100 independent measurements each for $o$ - and for $p$-xylene. The top graphic shows the histograms obtained using laser pulses with phase BP0; the bottom graphic shows the histograms obtained using laser pulses with phase BP858. The insets show the temporal profile of the two different laser pulses. The histograms obtained for BP858 show a 2.1 times greater molecular-ion-to-tropylium-ion ratio. Note that in most cases the distribution of measured values is compact, indicating good reproducibility of the results. The observed differences can be used for fast and reliable identification between these two isomers.

The time-frequency structure of the pulse may be responsible for the observed outcome of the reaction. We have yet to establish a direct link between the binary phase modulation of the pulse and the selective molecular response. However, the observed differences allowed us to devise a quantitative measurement of the relative concentration in a mixture of these isomers.

The quantitative performance of our method is demonstrated by the analysis of six different mixtures of the two xylene isomers. The mixtures were interrogated by the phase shaped pulses, and the ratio of the molecular ion to tropylium ion peaks was recorded. The results of two independent (different days) sets of measurements plotted as a function of concentration are shown in Figure 4 (top). The linear behavior of the data agrees with the analytical formula $\left(\mathrm{M}^{+} / \mathrm{T}^{+}\right)_{\text {normalized }}=\left[\left(\mathrm{M}^{+} / \mathrm{T}^{+}\right)_{\text {experimental }}\right.$ $\left.-\left(\mathrm{M}^{+} / \mathrm{T}^{+}\right)_{0}\right] /\left[\left(\mathrm{M}^{+} / \mathrm{T}^{+}\right)_{1}-\left(\mathrm{M}^{+} / \mathrm{T}^{+}\right)_{0}\right]$, where $\left(\mathrm{M}^{+} / \mathrm{T}^{+}\right)_{\text {experimental }}$ is the measured peak ratio for each sample mixture. $\left(\mathrm{M}^{+} / \mathrm{T}^{+}\right)_{0}$ is the peak ratio measured for a pure $p$-xylene sample, and $\left(\mathrm{M}^{+} /\right.$ $\left.\mathrm{T}^{+}\right)_{1}$ is the measured peak ratio for pure $o$-xylene. The normalized molecular-ion-to-tropylium ratios directly provide the relative concentration of the ortho isomer in the mixture.

Figure 4 (bottom) shows the maximum peak ratios obtained for the isomeric pairs of cresol, nitrotoluene (NT), 3-heptene, xylene, and 4-methyl-2-pentene (MP). We selected among the major fragment-ion peaks observed for each isomer pair to arrive at the reported ratios, thus ensuring a statistically acceptable standard deviation. Much greater ratios (in the hundreds) were observed when looking at minor peaks, but their usefulness for quantitative work is diminished by their greater susceptibility to noise.

With binary phase shaped femtosecond pulses, it is now possible to achieve subsecond, accurate, and quantitative

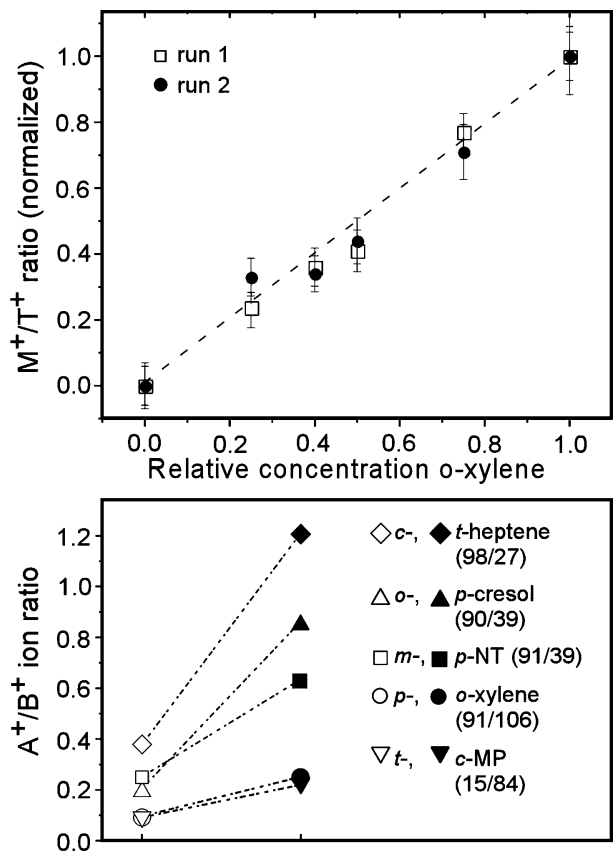

Figure 4. (top) Quantitative concentration measurement of isomeric mixtures using coherent laser control mass spectrometry. Each data point represents an average of 100 measurements $(0.1 \mathrm{~s}$ total $)$ obtained for each of six different mixtures. The horizontal axis corresponds to the known concentration of each of the mixtures. The vertical axis corresponds to the normalized $\mathrm{M}^{+} / \mathrm{T}^{+}$ratio, which increases linearly with the relative concentration of $o$-xylene. This linear relationship provides a quantitative method to determine the concentration of each of the isomers ( $o$ - and $p$-xylene) in a mixture. Two sets of data obtained in two different days are shown to illustrate the robustness of this method. (bottom) Ion peak ratios obtained for several isomeric pairs using binary shaped pulses. The legend indicates the $\mathrm{m} / \mathrm{z}$, value of the fragments chosen.

isomeric identification while maintaining the extremely high sensitivity of MS. This CLC-MS approach provides a new multidimensionality in MS that is highly sensitive to molecular structure, as required by the more recent biological applications of MS. A number of one-box, no-expert-required femtosecond lasers are already available that could be used for this purpose. Laser systems with integrated pulse shapers should be available by early 2006. At the fundamental level, our research demonstrates robust laser-based molecular recognition that can be improved severalfold by binary phase shaping. These results deserve further exploration at the experimental and theoretical levels.

Acknowledgment. We are grateful to Prof. J. T. Watson (MSU) for his suggestion to explore xylene identification. We thank Prof. G. Reid (MSU) for his perspective on our findings. We acknowledge the help of Dr. I. Pastirk of Biophotonic Solutions Inc. and Michael Kangas in the laser and pulse shaping systems and in the TOF-MS system, respectively. This research has been supported by CHE-0135581 of the National Science Foundation.

\section{References and Notes}

(1) Pastirk, I.; Kangas, M.; Dantus, M. J. Phys. Chem. A 2005, 109, 2413.

(2) Dantus, M.; Jannsen, M. H. M.; Zewail, A. H. Chem. Phys. Lett. 1991, 181, 281.

(3) Baumert, T.; Buhler, B.; Thalweiser, R.; Gerber, G. Phys. Rev. Lett. 1990, 64, 733.

(4) Assion, A.; Baumert, T.; Bergt, M.; Brixner, T.; Kiefer, B.; Seyfried, V.; Strehle, M.; Gerber, G. Science 1998, 282, 919. 
(5) Levis, R. J.; Menkir, G. M.; Rabitz, H. Science 2001, 292, 709.

(6) Weiner, A. M. Rev. Sci. Instrum. 2000, 71, 1929.

(7) Judson, R. S.; Rabitz, H. Phys. Rev. Lett. 1992, 68, 1500.

(8) Walowicz, K. A.; Pastirk, I.; Lozovoy, V. V.; Dantus, M. J. Phys. Chem. A 2002, 106, 9369.

(9) Lozovoy, V. V.; Pastirk, I.; Walowicz, K. A.; Dantus, M. J. Chem. Phys. 2003, 118, 3187.

(10) Comstock, M.; Lozovoy, V. V.; Pastirk, I.; Dantus, M. Opt. Express 2004, 12, 1061.

(11) A full account of the results obtained from these compounds is being prepared for publication elsewhere.

(12) Stein, S. E. In NIST Chemistry WebBook; NIST Standard Reference Database Number 69; Linstrom, P. J., Mallard, W. G., Eds.; National Institute of Standards and Technology: Gaithersburg, MD, 2005.

(13) Burlingame, A. L.; Shackleton, C. H. L.; Howe, I.; Chizhov, O. S Anal. Chem. 1978, 50, R346.

(14) McLafferty, F. W. Acc. Chem. Res. 1980, 13, 33.

(15) Bruckner, C. A.; Prazen, B. J.; Synovec, R. E. Anal. Chem. 1998, 70, 2796.

(16) Wu, H. F.; Chuan, Y. J. Rapid Commun. Mass Spectrom. 2003, 17, 1030 .

(17) Wu, H. F.; Wu, W. F. Rapid Commun. Mass Spectrom. 2003, 17 2399.

(18) Zimmermann, R.; Lerner, C.; Schramm, K.; Kettrup, A.; Boesl, U. Eur. J. Mass Spectrom. 1995, 1, 341

(19) Brodbelt, J. S.; Liou, C. C.; Donovan, T. Anal. Chem. 1991, 63 , 1205.
(20) Bjarnason, A. Anal. Chem. 1996, 68, 3882.

(21) Lozovoy, V. V.; Pastirk, I.; Dantus, M. Opt. Lett. 2004, 29, 775.

(22) Dela Cruz, J. M.; Pastirk, I.; Lozovoy, V. V.; Walowicz, K. A.; Dantus, M. J. Phys. Chem. A 2004, 108, 53.

(23) The phase shaped pulses were attenuated to $130 \mu \mathrm{J}$ and focused by a $50 \mathrm{~mm}$ lens onto the sample molecules. The samples $(o$ - and $p$-xylene, Sigma Aldrich) were used without further purification. The sample vapor was allowed to leak into the time-of-flight chamber until the pressure rose to $1 \times 10^{-5}$ Torr. The chamber was pumped by a $220 \mathrm{~L} / \mathrm{s}$ turbomolecular vacuum pump. Fast flow prevents the accumulation of fragments. Each mass spectrum was obtained after calibration of the arrival times at the detector and conversion of these times to the corresponding mass-to-charge ratio $(\mathrm{m} / \mathrm{z})$ and represents the signal average resulting from 128 laser shots $(\sim 0.1 \mathrm{~s})$.

(24) Malow, M.; Penno, M.; Weitzel, K. M. J. Phys. Chem A 2003 , 107,10625

(25) The binary phases are referred to in the text as BPX, where $X$ corresponds to the decimal value of the binary number; that is, the phase function 0000000000 corresponds to BP0 and 1101011010 corresponds to BP858, where 1 is replaced by $\pi$ retardation at the corresponding position in the spectrum.

(26) Lezius, M.; Blanchet, V.; Ivanov, M. Y.; Stolow, A. J. Chem. Phys. 2002, 117, 1575 .

(27) Smith, S. M.; Markevitch, A. N.; Romanov, D. A.; Li, X. S.; Levis, R. J.; Schlegel, H. B. J. Phys. Chem A 2004, 108, 11063. 